\title{
MULTIVARIATE GENERAL SPATIAL THREE-STAGE LEAST SQUARES FIXED EFFECT PANEL SIMULTANEOUS MODELS AND ESTIMATION OF THEIR PARAMETERS
}

\author{
TIMBANG SIRAIT \\ Program Studi Statistika \\ Politeknik Statistika STIS \\ Jalan Otto Iskandardinata No. 64C, Jakarta Timur, DKI Jakarta, 13330 \\ INDONESIA
}

\begin{abstract}
Simultaneous equation models describe a two-way flow of influence among variables. Simultaneous equation models using panel data, especially for fixed effect where there are spatial autoregressive and spatial errors with exact solutions, still require to be developed. In this paper, we develop the new models that it consist of spatial autoregressive and spatial errors. We call it as general spatial. This paper proposes feasible generalized least squares-three-stage least squares (FGLS-3SLS) to find all the estimators with exact solution and the numerical approximation estimators by concentrated log-likelihood formulation with method of forming sequence. All proposed estimators especially for closed-form estimators are proved to be consistent.
\end{abstract}

Key-Words: spatial autoregressive, spatial error, general spatial, FGLS-3SLS, concentrated log-likelihood, consistent

Received: January 3, 2020. Revised: June 13, 2020. Accepted: July 19, 2020. Published: August 8, 2020.

\section{Introduction}

If the model contains spatial influence and the spatial influence comes only through the error terms, we can use spatial error model [1]. Moreover, if the model contains spatial influence and the spatial influence comes only through the dependent, we can use spatial autoregressive model [2]. Now, we develop the paper where there are spatial autoregressive and spatial errors. The new models include two spatials, namely spatial autoregressive and spatial error. We call it general spatial.

System methods are the methods which are much more efficient than the single-equation methods because they use much more informations [3]. Single-equation methods and system methods are two methods which it can be used to find the estimators of parameter in simultaneous equation models [3].

Estimators of three-stage least squares (3SLS) are more robust than other estimators, like full information maximum likelihood (FIML) [4]. Consequently, solution technique by means of 3SLS is much more advantageous than the one by FIML because it is both time saving and cost saving.

In this solution, we still use first-order queen contiguity to find row-standardized spatial weight matrix [5] and Moran Index to examine spatial influence [6-8]. Some papers about estimation of parameter in simultaneous equation models for fixed effect are revealed in [9], and [10]. But, estimating these parameters had done by simulation.

In this paper, we are motivated to develop simultaneous equation models for fixed effect panel data with one-way error component by means of 3SLS solutions, especially for both spatial correlation among dependent variables and spatial correlation among errors. We call it as general spatial.

The objective of this paper is to obtain the closed-form estimators of parameter models by means of feasible generalized least squares-threestage least squares (FGLS-3SLS) and the numerical approximation estimators of parameter models by means of concentrated log-likelihood formulation with method of forming sequence. And then, to prove their consistency, especially for closed-form estimators.

\section{Models Development}

We had an equation by [2], namely

$$
\mathbf{y}_{h j}=\mathbf{1} \mu_{h}+\mathbf{X}_{h j} \boldsymbol{\alpha}_{h}+\mathbf{Y}_{-h j} \boldsymbol{\beta}_{-h}+\mathbf{1} \gamma_{h j}+\mathbf{u}_{h j} \text {, }
$$

for $h=1,2,3, \cdots, m, \quad j=1,2,3, \cdots, T$, where $\mathbf{y}_{h j}$ denotes the $j$ th time period $h$ th endogenous vector, $\mathbf{X}_{h j}$ denotes the $j$ th time period $h$ th matrix including (for example $k_{h}$ ) exogenous variables, 
$\mathbf{Y}_{-h j}$ denotes the $j$ th time period $-h$ th matrix including endogenous explanatory variables except the $j$ th time period $h$ th endogenous explanatory variables, $\mu_{h}$ denotes the $h$ th mean parameter, $\boldsymbol{\alpha}_{h}$ denotes the $h$ th parameters vector of exogenous variables, $\boldsymbol{\beta}_{-h}$ denotes the $-h$ th parameters vector of endogenous explanatory variables, $\gamma_{h j}$ denotes the $j$ th time period $h$ th time specific effect parameter, 1 denotes the unit vector, $\mathbf{u}_{h j}$ denotes the $j$ th time period $h$ th random error vector assuming mean vector $\mathbf{0}$ and covariance matrix $\sigma_{h}^{2} \mathbf{I}_{n}$ (homoscedasticity) in which $\sigma_{h}^{2}$ denotes the unknown $h$ th error variance and $\mathbf{I}_{n}$ denotes the $n \times n$ identity matrix. There is one restriction, namely $\sum_{j=1}^{T} \gamma_{h j}=0$. In this context, we suppose that (1) are over identified.

The next model is general spatial model (GSM) which refers to [11], namely:

$$
\begin{aligned}
& \mathbf{y}=\mathbf{1} \mu+\mathbf{X} \boldsymbol{\alpha}+\rho \mathbf{W} \mathbf{y}+\mathbf{u}, \\
& \mathbf{u}=\lambda \mathbf{W u}+\boldsymbol{\varepsilon}, \boldsymbol{\varepsilon} \square N\left(\mathbf{0}, \sigma^{2} \mathbf{I}_{n}\right),
\end{aligned}
$$

where $\mathbf{y}$ denotes the endogenous vector, $\mathbf{X}$ denotes the matrix of observations including (for example $k$ ) exogenous variables, $\mu$ denotes the mean parameter, $\boldsymbol{\alpha}$ denotes the parameters vector of exogenous variables, $\rho$ and $\lambda$ denote the spatial autoregressive and the spatial autocorrelation parameters, respectively, $\mathbf{W}$ denotes the rowstandardized spatial weight matrix, and $\mathbf{u}$ denotes the spatial autocorrelation of random error vector, and $\boldsymbol{\varepsilon}$ denotes the random error vector assuming normal distribution with mean vector $\mathbf{0}$ and covariance matrix $\sigma^{2} \mathbf{I}_{n}$ in which $\sigma^{2}$ denotes the unknown error variance.

If (1) contains spatial influences and the spatial influences come through the endogenous and the error variables, then we can adopt models in equations (2) and obtain new form equations as follows:

$$
\begin{aligned}
& \mathbf{y}_{h j}=\mathbf{1} \mu_{h}+\mathbf{X}_{h j} \boldsymbol{\alpha}_{h}+\rho_{h} \mathbf{W} \mathbf{y}_{h j}+\mathbf{Y}_{-h j} \boldsymbol{\beta}_{-h}+\mathbf{1} \gamma_{h j}+\mathbf{u}_{h j} \\
& \mathbf{u}_{h j}=\lambda_{h} \mathbf{W} \mathbf{u}_{h j}+\boldsymbol{\varepsilon}_{h j}, \boldsymbol{\varepsilon}_{h j} \square N\left(\mathbf{0}, \sigma_{h}^{2} \mathbf{I}_{n}\right) .
\end{aligned}
$$

Equation (3) can be simplified as follows:

$$
\mathbf{A}_{h} \mathbf{y}_{h j}=\mathbf{1} \mu_{h}+\mathbf{X}_{h j} \boldsymbol{\alpha}_{h}+\mathbf{Y}_{-h j} \boldsymbol{\beta}_{-h}+\mathbf{1} \gamma_{h j}+\mathbf{B}_{h}^{-1} \boldsymbol{\varepsilon}_{h j},
$$

for $\quad h=1,2,3, \cdots, m, \quad j=1,2,3, \cdots, T$, where $\mathbf{A}_{h}=\mathbf{I}_{n}-\rho_{h} \mathbf{W}, \mathbf{B}_{h}=\mathbf{I}_{n}-\lambda_{h} \mathbf{W}, \rho_{h}$ and $\lambda_{h}$ denote the $h$ th spatial autoregressive and the $h$ th spatial autocorrelation parameters, respectively, and $\mathbf{u}_{h j}$ denotes the $j$ th time period $h$ th spatial autocorrelation of random error vector, and $\boldsymbol{\varepsilon}_{h j}$ denotes the $j$ th time period $h$ th random error vector assuming normal distribution with mean vector $\mathbf{0}$ and covariance matrix $\sigma_{h}^{2} \mathbf{I}_{n}$. There is one restriction, namely $\sum_{j=1}^{T} \gamma_{h j}=0$.

We refer to [12] for the properties of kronecker products, [13] for reparameterization, [3, 4, 14] for 3SLS estimation, [15] for GLS and FGLS, [5] for the use first-order queen contiguity to find the rowstandardized spatial weight matrix, [6-8] for examining spatial influences by means of Moran Index and [16] for consistency.

For the solution of (4) by 3SLS, we obtain the following equation:

$$
\begin{aligned}
\overline{\mathbf{X}}_{*}^{t} \mathbf{A}_{h} \mathbf{Y}_{h j}= & \overline{\mathbf{X}}_{*}^{t} \mathbf{1} \mu_{h}+\overline{\mathbf{X}}_{*}^{t} \mathbf{X}_{h j} \boldsymbol{\alpha}_{h}+\overline{\mathbf{X}}_{*}^{t} \mathbf{Y}_{-h j} \boldsymbol{\beta}_{-h} \\
& +\overline{\mathbf{X}}_{*}^{t} \mathbf{1} \gamma_{h j}+\overline{\mathbf{X}}_{*}^{t} \mathbf{B}_{h}^{-1} \boldsymbol{\varepsilon}_{h j} .
\end{aligned}
$$

We use average value approach of the matrix of observations $[1,17,18]$ because the estimator of the mean is unbiased, consistent, and efficient as revealed by $[3,4,19]$. If we use $\mathbf{X}_{*_{j}}^{t}$ then the restriction $\sum_{j=1}^{T} \gamma_{h j}=0$ will not be achieved. This is due to $\mathbf{X}_{*_{j}}^{t}$ having in general, different values of the matrix of observations in every $j$ th time period $[1,7$, $17,18]$.

We can rewrite (5) to obtain new forms of vectors and matrices as follows:

$$
\overline{\mathbf{X}}_{* *}^{t} \mathbf{A} \mathbf{y}_{j}=\overline{\mathbf{X}}_{* *}^{t} \mathbf{G} \boldsymbol{\mu}+\overline{\mathbf{X}}_{* *}^{t} \mathbf{Z}_{j} \boldsymbol{\theta}+\overline{\mathbf{X}}_{* *}^{t} \mathbf{G} \boldsymbol{\gamma}_{j}+\overline{\mathbf{X}}_{* *}^{t} \mathbf{B}_{*} \boldsymbol{\varepsilon}_{j},
$$

where $\mathbf{Z}_{j}=\left[\begin{array}{lll}\mathbf{X}_{j} & \vdots & \mathbf{Y}_{-j}\end{array}\right]$ and $\boldsymbol{\theta}^{t}=\left[\begin{array}{lll}\boldsymbol{\alpha}^{t} & \vdots & \boldsymbol{\beta}_{-}^{t}\end{array}\right]$ having dimensions $m n \times\left(\sum_{h=1}^{m} k_{h}+m(m-1)\right)$ and $\left(\sum_{h=1}^{m} k_{h}+m(m-1)\right) \times 1$, respectively.

Explanation of the vectors and matrices from equations (5)-(6) are $\overline{\mathbf{X}}_{* *}$ denotes the $m n \times m \sum_{h=1}^{m} k_{h}$ diagonal matrix whose submain diagonal is $\overline{\mathbf{X}}_{*}$, $\overline{\mathbf{X}}_{*}=\frac{1}{T} \sum_{j=1}^{T} \mathbf{X}_{* j} \quad$ where $\quad \mathbf{X}_{*}$ denotes the $n \times \sum_{h=1}^{m} k_{h}$ matrix including all the exogenous variables in the system, A denotes the $m n \times m n$ diagonal matrix whose submain diagonal is the $n \times n$ matrix $\mathbf{A}_{h}$, $\mathbf{y}_{j}$ denotes the $m n \times 1$ vector including all of the $n \times 1$ vectors $\mathbf{y}_{h j}, \mathbf{G}$ denotes the $m n \times m$ diagonal 
matrix whose submain diagonal is $\mathbf{1}, \mathbf{B}_{*}$ denotes the $m n \times m n$ diagonal matrix whose submain diagonal is the $n \times n$ matrix $\mathbf{B}_{h}^{-1}, \boldsymbol{\mu}$ denotes the $m \times 1$ vector including all of $\mu_{h}, \mathbf{X}_{j}$ denotes the $m n \times \sum_{h=1}^{m} k_{h}$ diagonal matrix whose submain diagonal is the $n \times k_{h}$ matrix $\mathbf{X}_{h j}, \quad \boldsymbol{\alpha}$ denotes the $\sum_{h=1}^{m} k_{h} \times 1$ vector including all of the $k_{h} \times 1$ vectors $\boldsymbol{\alpha}_{h}, \mathbf{Y}_{-j}$ denotes the $m n \times m(m-1)$ diagonal matrix whose submain diagonal is the $n \times(m-1)$ matrix $\mathbf{Y}_{-h j}, \boldsymbol{\beta}_{-}$ denotes the $m(m-1) \times 1$ vector including all of the $(m-1) \times 1$ vectors $\boldsymbol{\beta}_{-h}, \boldsymbol{\gamma}_{j}$ denotes the $m \times 1$ vector including all of $\gamma_{h j}$, and $\boldsymbol{\varepsilon}_{j}$ denotes the $m n \times 1$ vector including all of the $n \times 1$ vectors $\boldsymbol{\varepsilon}_{h j}$, as well as $n$ denotes the sampel size of observations. For $j=1,2,3, \cdots, T$, the restriction $\sum_{j=1}^{T} \gamma_{h j}=0$ is changed $\sum_{j=1}^{T} \gamma_{j}=\mathbf{0}$

\section{Estimating the Parameters}

Now, we consider the equation (6). Estimation of parameters models are conducted in three stages. At the first-stage, we estimate all the endogenous explanatory variables in the system in every time period. This first-stage is the same as the previous paper [2].

At the second-stage, we estimate parameters of $\mu_{h}, \boldsymbol{\alpha}_{h}, \boldsymbol{\beta}_{-h}$, and $\gamma_{h j}$ to obtain residual estimate of equation (4). Because equation (4) has nonhomoscedastic error, we first tranform this equation. $\operatorname{var}\left(\mathbf{B}_{h}^{-1} \boldsymbol{\varepsilon}_{h j}\right)=\sigma_{h}^{2}\left(\mathbf{B}_{h}^{t} \mathbf{B}_{h}\right)^{-1} \neq \sigma_{h}^{2} \mathbf{I}_{n} . \quad$ Premultiplying

(4) by $\sigma_{h}^{-1}\left(\mathbf{B}_{h}^{t} \mathbf{B}_{h}\right)^{\frac{1}{2}}$, we obtain

$$
\begin{aligned}
\left(\mathbf{B}_{h}^{t} \mathbf{B}_{h}\right)^{\frac{1}{2}} \mathbf{A}_{h} \mathbf{y}_{h j}= & \left(\mathbf{B}_{h}^{t} \mathbf{B}_{h}\right)^{\frac{1}{2}} \mathbf{1}_{h}+\left(\mathbf{B}_{h}^{t} \mathbf{B}_{h}\right)^{\frac{1}{2}} \mathbf{X}_{h j} \boldsymbol{\alpha}_{h} \\
& +\left(\mathbf{B}_{h}^{t} \mathbf{B}_{h}\right)^{\frac{1}{2}} \mathbf{Y}_{-h j} \mathbf{B}_{-h}+\left(\mathbf{B}_{h}^{t} \mathbf{B}_{h}\right)^{\frac{1}{2}} \mathbf{1} \gamma_{h j}(7) \\
& +\left(\mathbf{B}_{h}^{t} \mathbf{B}_{h}\right)^{\frac{1}{2}} \mathbf{B}_{h}^{-1} \boldsymbol{\varepsilon}_{h j} .
\end{aligned}
$$

We can omit the value of $\sigma_{h}^{-1}$ because it is a finite constant. Now, the equation (7) has satisfied a regression model requirement.
We then substitute $\mathbf{Y}_{-h j}$ by $\hat{\mathbf{Y}}_{-h j}$ in (7), where $\mathbf{Y}_{-h j}=\hat{\mathbf{Y}}_{-h j}+\hat{\mathbf{V}}_{-h j}$, where $\hat{\mathbf{V}}_{-h j}$ is residual estimate from the first stage, and obtain new equations as follows:

$$
\begin{aligned}
\left(\mathbf{B}_{h}^{t} \mathbf{B}_{h}\right)^{\frac{1}{2}} \mathbf{A}_{h} \mathbf{y}_{h j}= & \left(\mathbf{B}_{h}^{t} \mathbf{B}_{h}\right)^{\frac{1}{2}} \mathbf{1} \mu_{h}+\left(\mathbf{B}_{h}^{t} \mathbf{B}_{h}\right)^{\frac{1}{2}} \mathbf{Z}_{h j} \boldsymbol{\theta}_{h} \\
& +\left(\mathbf{B}_{h}^{t} \mathbf{B}_{h}\right)^{\frac{1}{2}} \mathbf{1} \gamma_{h j}+\mathbf{u}_{h j}^{*},
\end{aligned}
$$

where $\mathbf{Z}_{h j}=\left[\begin{array}{lll}\mathbf{X}_{h j} & \vdots & \hat{\mathbf{Y}}_{-h j}\end{array}\right]$ and $\boldsymbol{\theta}_{h}^{t}=\left[\begin{array}{lll}\boldsymbol{\alpha}_{h}^{t} & \vdots & \boldsymbol{\beta}_{-h}^{t}\end{array}\right]$ having dimensions $n \times\left(k_{h}+m-1\right)$ and $1 \times\left(k_{h}+m-1\right)$, respectively, and $\mathbf{u}_{h j}^{*}$ denotes the composite random error with $\mathbf{u}_{h j}^{*}=\left(\mathbf{B}_{h}^{t} \mathbf{B}_{h}\right)^{\frac{1}{2}} \hat{\mathbf{V}}_{-h j} \boldsymbol{\beta}_{-h}+\left(\mathbf{B}_{h}^{t} \mathbf{B}_{h}\right)^{\frac{1}{2}} \mathbf{B}_{h}^{-1} \boldsymbol{\varepsilon}_{h j}$.

The right-hand side matrix of equation (8) is less than full rank. But, we can not use $n \times n$ dimensional transformation matrix $\mathbf{Q}$ directly, in which $\mathbf{Q 1}=\mathbf{0}$, to find the estimator of $\boldsymbol{\theta}_{h}$. We remind again that $\mathbf{Q}=\mathbf{I}_{n}-\frac{1}{n} \mathbf{1 1}^{t}$ is symmetrical and idempotent matrices.

We need to reparameterize equation (8), namely:

$$
\begin{aligned}
\left(\mathbf{B}_{h}^{t} \mathbf{B}_{h}\right)^{\frac{1}{2}} \mathbf{A}_{h} \mathbf{y}_{h j}= & \left(\mathbf{B}_{h}^{t} \mathbf{B}_{h}\right)^{\frac{1}{2}} \mathbf{1} \mu_{h j}+\left(\mathbf{B}_{h}^{t} \mathbf{B}_{h}\right)^{\frac{1}{2}} \mathbf{Z}_{h j} \boldsymbol{\theta}_{h} \\
& +\mathbf{u}_{h j}^{*},
\end{aligned}
$$

where $\mu_{h j}=\mu_{h}+\gamma_{h j}$.

By GLS Solution, we first get the estimator of $\mu_{h j}$, namely

$$
\hat{\mu}_{h j}=\left(\mathbf{1}^{t} \mathbf{B}_{h}^{t} \mathbf{B}_{h} \mathbf{1}\right)^{-1} \mathbf{1}^{t} \mathbf{B}_{h}^{t} \mathbf{B}_{h}\left(\mathbf{A}_{h} \mathbf{y}_{h j}-\mathbf{Z}_{h j} \boldsymbol{\theta}_{h}\right)
$$

We then use (10) to find estimator of $\boldsymbol{\theta}_{h}$ and by GLS solution, we obtain

$$
\begin{aligned}
\hat{\boldsymbol{\theta}}_{h}= & {\left[\sum_{j=1}^{T} \mathbf{Z}_{h j}^{t} \mathbf{B}_{h}^{t} \mathbf{B}_{h}\left[\mathbf{1 b}_{h}^{t}-\mathbf{I}_{n}\right] \mathbf{Z}_{h j}\right]^{-1} } \\
& \times \sum_{j=1}^{T} \mathbf{Z}_{h j}^{t} \mathbf{B}_{h}^{t} \mathbf{B}_{h}\left[\mathbf{1} \mathbf{b}_{h}^{t}-\mathbf{I}_{n}\right] \mathbf{A}_{h} \mathbf{y}_{h j},
\end{aligned}
$$

where $\mathbf{b}_{h}^{t}=\left(\mathbf{1}^{t} \mathbf{B}_{h}^{t} \mathbf{B}_{h} \mathbf{1}\right)^{-1} \mathbf{1}^{t} \mathbf{B}_{h}^{t} \mathbf{B}_{h}$ having dimension $1 \times n$.

We recall to equation (8) and use the GLS solution, we obtain the estimators of $\mu_{h}$ and $\gamma_{h j}$, namely

$$
\hat{\mu}_{h}=\frac{1}{T} \mathbf{b}_{h}^{t} \sum_{j=1}^{T}\left(\mathbf{A}_{h} \mathbf{y}_{h j}-\mathbf{Z}_{h j} \hat{\boldsymbol{\theta}}_{h}\right),
$$

and

$$
\hat{\gamma}_{h j}=\mathbf{b}_{h}^{t}\left(\mathbf{A}_{h} \mathbf{y}_{h j}-\mathbf{1} \hat{\mu}_{h}-\mathbf{Z}_{h j} \hat{\boldsymbol{\theta}}_{h}\right) .
$$

respectively. 
From (11) to (13), we can estimate $\mathbf{u}_{h j}^{*}$ as follows $\hat{\mathbf{u}}_{h j}^{*}=\mathbf{A}_{h} \mathbf{y}_{h j}-\mathbf{1}\left(\hat{\mu}_{h}+\hat{\gamma}_{h j}\right)-\mathbf{Z}_{h j} \hat{\boldsymbol{\theta}}_{h}=\mathbf{A}_{h} \mathbf{y}_{h j}-\hat{\mathbf{a}}_{h j}$.

Matrices of $\mathbf{A}_{h}$ and $\mathbf{B}_{h}$ contain $\rho_{h}$ and $\lambda_{h}$. In case $\rho_{h}$ and $\lambda_{h}$ are not known, we can estimate it by means of concentrated log-likelihood.

We pay attention to equation (4). Premultiplying its both sides by $\mathbf{B}_{h}$, we obtain

$$
\begin{aligned}
\mathbf{B}_{h} \mathbf{A}_{h} \mathbf{y}_{h j}= & \mathbf{B}_{h} \mathbf{1} \mu_{h}+\mathbf{B}_{h} \mathbf{X}_{h j} \boldsymbol{\alpha}_{h}+\mathbf{B}_{h} \mathbf{Y}_{-h j} \boldsymbol{\beta}_{-h} \\
& +\mathbf{B}_{h} \mathbf{1} \gamma_{h j}+\boldsymbol{\varepsilon}_{h j},
\end{aligned}
$$

By equation (15), the likelihood function of $\boldsymbol{\varepsilon}_{h j}$, $j=1,2,3, \cdots, T$, denoted by $L_{h}$ is as follows: $L_{h}=\prod_{j=1}^{T}\left(2 \pi \sigma_{h}^{2}\right)^{-\frac{n}{2}} \exp \left(-\frac{1}{2 \sigma_{h}^{2}} \boldsymbol{\varepsilon}_{h j}^{t} \boldsymbol{\varepsilon}_{h j}\right), \quad$ and by Jacobian transformation, we obtain the natural logarithm of $L_{h}$ as follow:

$$
\begin{aligned}
\ln L_{h}= & -\frac{n T}{2} \ln \left(2 \pi \sigma_{h}^{2}\right)-\frac{1}{2 \sigma_{h}^{2}} \sum_{j=1}^{T}\left(\mathbf{B}_{h} \mathbf{A}_{h} \mathbf{y}_{h j}-\mathbf{B}_{h} \mathbf{a}_{h j}\right)^{t} \\
& \times\left(\mathbf{B}_{h} \mathbf{A}_{h} \mathbf{y}_{h j}-\mathbf{B}_{h} \mathbf{a}_{h j}\right)+T \ln \left\|\mathbf{B}_{h}\right\|+T \ln \left\|\mathbf{A}_{h}\right\|,
\end{aligned}
$$

where $\left\|\mathbf{A}_{h}\right\|$ and $\left\|\mathbf{B}_{h}\right\|$ are the absolute of the determinants of $\mathbf{A}_{h}$ and of $\mathbf{B}_{h}$, respectively.

We take derivative for $\sigma_{h}^{2}$. Setting this derivative equal to zero, we obtain the estimator of $\sigma_{h}^{2}$, namely:

$$
\begin{aligned}
\hat{\sigma}_{h}^{2}= & \frac{1}{n T} \sum_{j=1}^{T}\left(\mathbf{B}_{h} \mathbf{A}_{h} \mathbf{y}_{h j}-\mathbf{B}_{h} \mathbf{a}_{h j}\right)^{t} \\
& \left(\mathbf{B}_{h} \mathbf{A}_{h} \mathbf{y}_{h j}-\mathbf{B}_{h} \mathbf{a}_{h j}\right) .
\end{aligned}
$$

By (18), we obtain concentrated log-likelihood as follows:

$$
\begin{aligned}
\ln L_{h}^{c o n}= & C-\frac{n T}{2} \ln \left(\frac{1}{n T} \sum_{j=1}^{T}\left(\mathbf{B}_{h} \mathbf{A}_{h} \mathbf{y}_{h j}-\mathbf{B}_{h} \mathbf{a}_{h j}\right)^{t}\right. \\
& \left.\times\left(\mathbf{B}_{h} \mathbf{A}_{h} \mathbf{y}_{h j}-\mathbf{B}_{h} \mathbf{a}_{h j}\right)\right)+T \ln \left\|\mathbf{B}_{h}\right\| \\
& +T \ln \left\|\mathbf{A}_{h}\right\|,
\end{aligned}
$$

where $C=-\frac{n T}{2} \ln (2 \pi)-\frac{n T}{2}$.

Let $\mathbf{W}$ have eigenvalues $\omega_{1}, \omega_{2}, \cdots, \omega_{n}$. The acceptable spatial autoregressive and spatial autocorrelation parameters are $\frac{1}{\omega_{\text {minimum }}}<\rho_{h}\left(\lambda_{h}\right)<1$ [20]. We use numerical method for $\ln L_{h}^{c o n}$ to find estimators of $\rho_{h}$ and $\lambda_{h}$, namely method of forming sequence of $\rho_{h}$ and $\lambda_{h}$ by means of R program [1, $7,17,18]$. Its procedure is as follows:

1. We make sequences values of $\rho_{h}$ and $\lambda_{h}$, respectively, namely $\rho_{h}=$ seq(start value, end value, increasing), and $\lambda_{h}=$ seq(start value, end value, increasing).

2. For every $\mathbf{y}_{h j}$ and $\mathbf{a}_{h j}, h=1,2,3, \cdots m$, we insert values of $\rho_{h}$ and $\lambda_{h}$ in (17). Because the values of $\mathbf{a}_{h j}$ are unknown, we use the estimator, $\hat{\mathbf{a}}_{h j}$, where $\quad \hat{\mathbf{a}}_{h j}=\mathbf{1}\left(\hat{\mu}_{h}+\hat{\gamma}_{h j}\right)+\mathbf{Z}_{h j} \hat{\boldsymbol{\theta}}_{h}, \quad$ with $\mathbf{Z}_{h j}=\left[\begin{array}{lll}\mathbf{X}_{h j} & \vdots & \hat{\mathbf{Y}}_{-h j}\end{array}\right]$.

3. Finding the values of $\rho_{h}$ and $\lambda_{h}$ that gives the largest $\ln L_{h}^{c o n}$.

Based on the estimate $\rho_{h}$ and $\lambda_{h}$, the equations (11) to (13) can be rewritten as follows:

$$
\begin{aligned}
\hat{\boldsymbol{\theta}}_{h}= & {\left[\sum_{j=1}^{T} \mathbf{Z}_{h j}^{t} \hat{\mathbf{B}}_{h}^{t} \hat{\mathbf{B}}_{h}\left[\mathbf{1} \mathbf{b}_{h}^{t}-\mathbf{I}_{n}\right] \mathbf{Z}_{h j}\right]^{-1} } \\
& \times \sum_{j=1}^{T} \mathbf{Z}_{h j}^{t} \hat{\mathbf{B}}_{h}^{t} \hat{\mathbf{B}}_{h}\left[\mathbf{1} \mathbf{b}_{h}^{t}-\mathbf{I}_{h}\right] \hat{\mathbf{A}}_{h} \mathbf{y}_{h j}, \\
\hat{\mu}_{h}= & \frac{1}{T} \mathbf{b}_{h}^{t} \sum_{j=1}^{T}\left(\hat{\mathbf{A}}_{h} \mathbf{y}_{h j}-\mathbf{Z}_{h j} \hat{\boldsymbol{\theta}}_{h}\right),
\end{aligned}
$$

and

$$
\hat{\gamma}_{h j}=\mathbf{b}_{h}^{t}\left(\hat{\mathbf{A}}_{h} \mathbf{y}_{h j}-\mathbf{1} \hat{\mu}_{h}-\mathbf{Z}_{h j} \hat{\boldsymbol{\theta}}_{h}\right),
$$

respectively, where

$\hat{\mathbf{A}}_{h}=\mathbf{I}_{n}-\hat{\rho}_{h} \mathbf{W}, \hat{\mathbf{B}}_{h}=\mathbf{I}_{n}-\hat{\lambda}_{h} \mathbf{W}$, and

$\mathbf{b}_{h}^{t}=\left(\mathbf{1}^{t} \hat{\mathbf{B}}_{h}^{t} \hat{\mathbf{B}}_{h} \mathbf{1}\right)^{-1} \mathbf{1}^{t} \hat{\mathbf{B}}_{h}^{t} \hat{\mathbf{B}}_{h}$.

The furthermore, equation (14) can be rewritten as follows:

$\hat{\mathbf{u}}_{h j}^{*}=\hat{\mathbf{A}}_{h} \mathbf{y}_{h j}-\mathbf{1}\left(\hat{\mu}_{h}+\hat{\gamma}_{h j}\right)-\mathbf{Z}_{h j} \hat{\boldsymbol{\theta}}_{h}=\hat{\mathbf{A}}_{h} \mathbf{y}_{h j}-\hat{\mathbf{a}}_{h j}$.

We then use (21) and (16) to find the estimated covariance matrix of the estimator $\hat{\mathbf{u}}_{h j}^{*}$, namely

$$
\hat{\boldsymbol{\Sigma}}=\left[\begin{array}{ccccc}
\hat{\sigma}_{1}^{2} & \hat{\sigma}_{12} & \hat{\sigma}_{13} & \cdots & \hat{\sigma}_{1 m} \\
\hat{\sigma}_{21} & \hat{\sigma}_{2}^{2} & \hat{\sigma}_{23} & \cdots & \hat{\sigma}_{2 m} \\
\hat{\sigma}_{31} & \hat{\sigma}_{32} & \hat{\sigma}_{3}^{2} & \cdots & \hat{\sigma}_{3 m} \\
\vdots & \vdots & \vdots & \ddots & \vdots \\
\hat{\sigma}_{m 1} & \hat{\sigma}_{m 2} & \hat{\sigma}_{m 3} & \cdots & \hat{\sigma}_{m}^{2}
\end{array}\right], \hat{\sigma}_{h}^{2}=\hat{\sigma}_{h h^{*}} \text { if } h=h^{*}
$$

with

$\hat{\sigma}_{h h^{*}}=\frac{1}{T(n-1)-(k+m-1)} \sum_{j=1}^{T} \hat{\mathbf{u}}_{h j}^{* t} \hat{\mathbf{B}}_{h}^{t} \hat{\mathbf{B}}_{h^{\prime}} \hat{\mathbf{u}}_{h^{*} j}^{*}$,

where $\hat{\sigma}_{h}^{2}$ denotes the $h$ th estimated error variance, $\hat{\sigma}_{h h^{*}}$ denotes the $h^{*}$ th and the $h$ th estimated error covariance, and $\hat{\boldsymbol{\Sigma}}$ denotes $m \times m$ estimated covariance matrix. We change the denominator of (16) so that it becomes an unbiased estimator [18]. 
From (6), we have an error covariance matrix, namely $\operatorname{var}\left(\overline{\mathbf{X}}_{* *}^{t} \hat{\mathbf{B}}_{*} \boldsymbol{\varepsilon}_{j}\right)=\overline{\mathbf{X}}_{* *}^{t} \hat{\mathbf{B}}_{*} \operatorname{var}\left(\boldsymbol{\varepsilon}_{j}\right) \hat{\mathbf{B}}_{*}^{t} \overline{\mathbf{X}}_{* *}=\boldsymbol{\Sigma}_{\#}$. This covariance shows that the random errors are heteroscedastic, where $\operatorname{var}\left(\boldsymbol{\varepsilon}_{j}\right)=E\left(\boldsymbol{\varepsilon}_{j} \boldsymbol{\varepsilon}_{j}^{t}\right)$ for $h=h^{*}=1,2,3, \cdots, m$,

$\boldsymbol{\varepsilon}_{j}^{t}=\left[\begin{array}{lllll}\boldsymbol{\varepsilon}_{1 j}^{t} & \boldsymbol{\varepsilon}_{2 j}^{t} & \boldsymbol{\varepsilon}_{3 j}^{t} & \cdots & \boldsymbol{\varepsilon}_{m j}^{t}\end{array}\right]$,

$\boldsymbol{\varepsilon}_{h j}^{t}=\left[\begin{array}{lllll}\varepsilon_{h 1 j} & \varepsilon_{h 2 j} & \varepsilon_{h 3 j} & \cdots & \varepsilon_{h n j}\end{array}\right]$,

in which we assumed that

$E\left(\varepsilon_{h i j} \varepsilon_{h^{*} i^{*} j}\right)= \begin{cases}\sigma_{h h^{*}} & \text { if } i=i^{*} \\ 0 & \text { if } i \neq i^{*}\end{cases}$

so that $E\left(\boldsymbol{\varepsilon}_{h j} \boldsymbol{\varepsilon}_{h^{*} j}^{t}\right)=\sigma_{h h^{\prime}} \mathbf{I}_{n}$. We obtain $\operatorname{var}\left(\boldsymbol{\varepsilon}_{j}\right)=\boldsymbol{\Sigma} \otimes \mathbf{I}_{n}$ with $m n \times m n$ as its dimension. Consequently, $\boldsymbol{\Sigma}_{\#}=\overline{\mathbf{X}}_{* *}^{t} \hat{\mathbf{B}}_{*}\left(\boldsymbol{\Sigma} \otimes \mathbf{I}_{n}\right) \hat{\mathbf{B}}_{*}^{t} \overline{\mathbf{X}}_{* *} \quad$ which is $m \sum_{h=1}^{m} k_{h} \times m \sum_{h=1}^{m} k_{h} \quad$ symmetrical matrix. If $\boldsymbol{\Sigma}$ is unknown then we can use its estimator. If we use its estimator then $\hat{\boldsymbol{\Sigma}}_{\#}=\overline{\mathbf{X}}_{* *}^{t} \hat{\mathbf{B}}_{*}\left(\hat{\boldsymbol{\Sigma}} \otimes \mathbf{I}_{n}\right) \hat{\mathbf{B}}_{*}^{t} \overline{\mathbf{X}}_{* *}$.

In the above results, we see that the error variance in equation (6) is not constant and the matrix in the right-hand side is less than full rank. For the last-stage, we overcome those problems again by means of reparameterization and GLS. The estimators are as follows:

$$
\begin{aligned}
\hat{\boldsymbol{\theta}} & =\left[\sum_{j=1}^{T} \mathbf{Z}_{j}^{t} \hat{\mathbf{H}}^{*} \hat{\mathbf{M}}^{*} \mathbf{Z}_{j}\right]^{-1} \sum_{j=1}^{T} \mathbf{Z}_{j}^{t} \hat{\mathbf{H}}^{*} \hat{\mathbf{M}}^{*} \hat{\mathbf{A}} \mathbf{y}_{j}, \\
\hat{\boldsymbol{\mu}} & =\left[T \mathbf{G}^{t} \hat{\mathbf{H}}^{*} \mathbf{G}\right]^{-1} \mathbf{G}^{t} \hat{\mathbf{H}}^{*} \sum_{j=1}^{T}\left(\hat{\mathbf{A}} \mathbf{y}_{j}-\mathbf{Z}_{j} \hat{\boldsymbol{\theta}}\right), \\
\hat{\boldsymbol{\gamma}}_{j} & =\left[\mathbf{G}^{t} \hat{\mathbf{H}}^{*} \mathbf{G}\right]^{-1} \mathbf{G}^{t} \hat{\mathbf{H}}^{*}\left(\hat{\mathbf{A}} \mathbf{y}_{j}-\mathbf{G} \hat{\boldsymbol{\mu}}-\mathbf{Z}_{j} \hat{\boldsymbol{\theta}}\right) .
\end{aligned}
$$

where

$\hat{\mathbf{H}}^{*}=\overline{\mathbf{X}}_{* *} \boldsymbol{\Sigma}_{\#}^{-1} \overline{\mathbf{X}}_{* *}^{t}$ and

$\hat{\mathbf{M}}^{*}=\mathbf{G}\left[\mathbf{G}^{t} \hat{\mathbf{H}}^{*} \mathbf{G}\right]^{-1} \mathbf{G}^{t} \hat{\mathbf{H}}^{*}-\mathbf{I}_{m n}$.

They have dimensions $m n \times m n$, respectively.

In this paper, the estimators of $\boldsymbol{\theta , \alpha}$, and $\boldsymbol{\gamma}_{j}$ are called the estimators of feasible generalized least squares-multivariate general spatial three-stage least squares fixed effect panel simultaneous models (FGLS-MGS3SLSFEPSM).

\section{Properties of Estimators}

Theorem (Consistency). If

$\overline{\mathbf{X}}_{* *}^{t} \mathbf{A} \mathbf{y}_{j}=\overline{\mathbf{X}}_{* *}^{t} \mathbf{G} \boldsymbol{\mu}+\overline{\mathbf{X}}_{* *}^{t} \mathbf{Z}_{j} \boldsymbol{\theta}+\overline{\mathbf{X}}_{* *}^{t} \mathbf{G} \boldsymbol{\gamma}_{j}+\overline{\mathbf{X}}_{* *}^{t} \mathbf{B}_{*} \boldsymbol{\varepsilon}_{j}$ as defined in (6), then $\hat{\boldsymbol{\theta}}, \hat{\boldsymbol{\mu}}$, and $\hat{\boldsymbol{\gamma}}_{j}$ are consistent estimators.

Proof. Recall (6). This can be rewritten as $\mathbf{A y}_{j}=\mathbf{G} \boldsymbol{\mu}+\mathbf{Z}_{j} \boldsymbol{\theta}+\mathbf{G} \boldsymbol{\gamma}_{j}+\mathbf{B}_{*} \boldsymbol{\varepsilon}_{j}$. However, we use the estimate $\hat{\rho}_{h}$ and $\hat{\lambda}_{h}$. The equation (6) can be rewritten as $\quad \hat{\mathbf{A}} \mathbf{y}_{j}=\mathbf{G} \boldsymbol{\mu}+\mathbf{Z}_{j} \boldsymbol{\theta}+\mathbf{G} \boldsymbol{\gamma}_{j}+\hat{\mathbf{B}}_{*} \boldsymbol{\varepsilon}_{j}$. Estimators of equation (6) are as follows:

$$
\begin{aligned}
\hat{\boldsymbol{\theta}} & =\left[\sum_{j=1}^{T} \mathbf{Z}_{j}^{t} \hat{\mathbf{H}}^{*} \hat{\mathbf{M}}^{*} \mathbf{Z}_{j}\right]^{-1} \sum_{j=1}^{T} \mathbf{Z}_{j}^{t} \hat{\mathbf{H}}^{*} \hat{\mathbf{M}}^{*} \hat{\mathbf{A}} \mathbf{y}_{j} \\
& =\boldsymbol{\theta}+\left[\sum_{j=1}^{T} \mathbf{Z}_{j}^{t} \hat{\mathbf{H}}^{*} \hat{\mathbf{M}}^{*} \mathbf{Z}_{j}\right]^{-1}\left[\sum_{j=1}^{T} \mathbf{Z}_{j}^{t} \hat{\mathbf{H}}^{*} \hat{\mathbf{M}}^{*} \hat{\mathbf{B}}_{*} \boldsymbol{\varepsilon}_{j}\right],
\end{aligned}
$$

where $\hat{\mathbf{M}}^{*} \mathbf{G}=\mathbf{0}$,

$$
\begin{aligned}
\hat{\boldsymbol{\mu}} & =\left[T \mathbf{G}^{t} \hat{\mathbf{H}}^{*} \mathbf{G}\right]^{-1} \mathbf{G}^{t} \hat{\mathbf{H}}^{*} \sum_{j=1}^{T}\left(\hat{\mathbf{A}} \mathbf{y}_{j}-\mathbf{Z}_{j} \hat{\boldsymbol{\theta}}\right) \\
& =\boldsymbol{\mu}+\left[T \mathbf{G}^{t} \hat{\mathbf{H}}^{*} \mathbf{G}\right]^{-1}\left(\sum_{j=1}^{T} \mathbf{G}^{t} \hat{\mathbf{H}}^{*} \mathbf{Z}_{j}(\boldsymbol{\theta}-\hat{\boldsymbol{\theta}})+\sum_{j=1}^{T} \mathbf{G}^{t} \hat{\mathbf{H}}^{*} \hat{\mathbf{B}}_{*} \boldsymbol{\varepsilon}_{j}\right),
\end{aligned}
$$

where $\sum_{j=1}^{T} \boldsymbol{\gamma}_{j}=\mathbf{0}$, and

$$
\begin{aligned}
\hat{\boldsymbol{\gamma}}_{j}= & {\left[\mathbf{G}^{t} \hat{\mathbf{H}}^{*} \mathbf{G}\right]^{-1} \mathbf{G}^{t} \hat{\mathbf{H}}^{*}\left(\hat{\mathbf{A}} \mathbf{y}_{j}-\mathbf{G} \hat{\boldsymbol{\mu}}-\mathbf{Z}_{j} \hat{\boldsymbol{\theta}}\right) } \\
= & (\boldsymbol{\mu}-\hat{\boldsymbol{\mu}})+\left[\mathbf{G}^{t} \hat{\mathbf{H}}^{*} \mathbf{G}\right]^{-1} \mathbf{G}^{t} \hat{\mathbf{H}}^{*} \mathbf{Z}_{j}(\boldsymbol{\theta}-\hat{\boldsymbol{\theta}})+\boldsymbol{\gamma}_{j} \\
& +\left[\mathbf{G}^{t} \hat{\mathbf{H}}^{*} \mathbf{G}\right]^{-1} \mathbf{G}^{t} \hat{\mathbf{H}}^{*} \hat{\mathbf{B}}_{*} \boldsymbol{\varepsilon}_{j} .
\end{aligned}
$$

We refer to $[3,4,14,16,21-23]$. Asymptotic expectation and variance of $\hat{\boldsymbol{\theta}}, \hat{\boldsymbol{\mu}}$, and $\hat{\boldsymbol{\gamma}}_{j}$ are as follows:

$$
\begin{aligned}
\bar{E}\{\hat{\boldsymbol{\theta}}\}= & \lim _{\substack{n \rightarrow \infty \\
T \rightarrow \infty}} E\{\hat{\boldsymbol{\theta}}\}=\boldsymbol{\theta}+\lim _{\substack{n \rightarrow \infty \\
T \rightarrow \infty}} E\left\{\left[\frac{1}{n T} \sum_{j=1}^{T} \mathbf{Z}_{j}^{t} \hat{\mathbf{H}}^{*} \hat{\mathbf{M}}^{*} \mathbf{Z}_{j}\right]^{-1}\right. \\
& \left.\times\left[\frac{1}{n T} \sum_{j=1}^{T} \mathbf{Z}_{j}^{t} \hat{\mathbf{H}}^{*} \hat{\mathbf{M}}^{*} \hat{\mathbf{B}}_{*} \boldsymbol{\varepsilon}_{j}\right]\right\} \\
= & \boldsymbol{\theta}+\left[\lim _{\substack{n \rightarrow \infty \\
T \rightarrow \infty}} \frac{1}{n T} \mathbf{K}\right]^{-1}\left[\lim _{\substack{n \rightarrow \infty \\
T \rightarrow \infty}} \frac{1}{n T} \sum_{j=1}^{T} \mathbf{Z}_{j}^{t} \hat{\mathbf{H}}^{*} \hat{\mathbf{M}}^{*} \hat{\mathbf{B}}_{*} \times \mathbf{0}\right] \\
& =\boldsymbol{\theta}+\left[\lim _{\substack{n \rightarrow \infty \\
T \rightarrow \infty}} \overline{\mathbf{K}}\right]^{-1} \times \mathbf{0}=\boldsymbol{\theta}+[\overline{\mathbf{K}}]^{-1} \times \mathbf{0} \\
& =\boldsymbol{\theta},
\end{aligned}
$$

where $\mathbf{K}$ and $\overline{\mathbf{K}}$ are constant nonsingular matrices. 
asy. $\operatorname{var}\{\hat{\boldsymbol{\theta}}\}=$ asy. var $\left\{\left[\sum_{j=1}^{T} \mathbf{Z}_{j}^{t} \hat{\mathbf{H}}^{*} \hat{\mathbf{M}}^{*} \mathbf{Z}_{j}\right]^{-1} \mathbf{Z}_{j}^{t} \hat{\mathbf{H}}^{*} \hat{\mathbf{M}}^{*} \hat{\mathbf{B}}_{*} \boldsymbol{\varepsilon}_{j}\right\}$ $=\left[\sum_{j=1}^{T} \mathbf{Z}_{j}^{t} \hat{\mathbf{H}}^{*} \hat{\mathbf{M}}^{*} \mathbf{Z}_{j}\right]^{-1}\left[\sum_{j=1}^{T} \mathbf{Z}_{j}^{t} \hat{\mathbf{H}}^{*} \hat{\mathbf{M}}^{*} \hat{\mathbf{B}}_{*}\left(\boldsymbol{\Sigma} \otimes \mathbf{I}_{n}\right)\right.$ $\left.\times \hat{\mathbf{B}}_{*}^{t} \hat{\mathbf{H}}^{*} \hat{\mathbf{M}}^{*} \mathbf{Z}_{j}\right]\left[\sum_{j=1}^{T} \mathbf{Z}_{j}^{t} \hat{\mathbf{H}}^{*} \hat{\mathbf{M}}^{*} \mathbf{Z}_{j}\right]^{-1}$,

where $\hat{\mathbf{H}}^{*}$ and $\hat{\mathbf{H}}^{*} \hat{\mathbf{M}}^{*}$ are symmetrical. Now, $\lim _{\substack{n \rightarrow \infty \\ T \rightarrow \infty}} \operatorname{asy} \cdot \operatorname{var}\{\hat{\boldsymbol{\theta}}\}=\left[\sum_{j=1}^{T} \mathbf{Z}_{j}^{t} \hat{\mathbf{H}}^{*} \hat{\mathbf{M}}^{*} \mathbf{Z}_{j}\right]^{-1}\left[\sum_{j=1}^{T} \mathbf{Z}_{j}^{t} \hat{\mathbf{H}}^{*} \hat{\mathbf{M}}^{*} \hat{\mathbf{B}}_{*}\right.$

$$
\begin{aligned}
& \left.\times\left\{\lim _{\substack{n \rightarrow \infty \\
T \rightarrow \infty}} \frac{1}{n T}\left(\boldsymbol{\Sigma} \otimes \mathbf{I}_{n}\right)\right\} \hat{\mathbf{B}}_{*}^{t} \hat{\mathbf{H}}^{*} \hat{\mathbf{M}}^{*} \mathbf{Z}_{j}\right] \\
& \times\left[\lim _{\substack{n \rightarrow \infty \\
T \rightarrow \infty}} \frac{1}{n T} \sum_{j=1}^{T} \mathbf{Z}_{j}^{t} \hat{\mathbf{H}}^{*} \hat{\mathbf{M}}^{*} \mathbf{Z}_{j}\right]^{-1}
\end{aligned}
$$

$\lim _{\substack{n \rightarrow \infty \\ T \rightarrow \infty}} \operatorname{asy} . \operatorname{var}\{\hat{\boldsymbol{\theta}}\}=[\mathbf{K}]^{-1}\left[\sum_{j=1}^{T} \mathbf{Z}_{j}^{t} \hat{\mathbf{H}}^{*} \hat{\mathbf{M}}^{*} \hat{\mathbf{B}}_{*} \times \mathbf{0}\right.$

$$
\begin{aligned}
& \left.\times \hat{\mathbf{B}}_{*}^{t} \hat{\mathbf{H}}^{*} \hat{\mathbf{M}}^{*} \mathbf{Z}_{j}\right]\left[\lim _{\substack{n \rightarrow \infty \\
T \rightarrow \infty}} \overline{\mathbf{K}}\right]^{-1} \\
= & \mathbf{K}^{-1} \times \mathbf{0} \times[\overline{\mathbf{K}}]^{-1}=\mathbf{0},
\end{aligned}
$$

This shows that $\hat{\boldsymbol{\theta}}$ is asymptotically unbiased estimator. If $n \rightarrow \infty$ or $T \rightarrow \infty$ or both of $n \rightarrow \infty$ and $T \rightarrow \infty$, then asy.var $\{\hat{\boldsymbol{\theta}}\} \rightarrow \mathbf{0}$. Therefore, $\hat{\boldsymbol{\theta}}$ is a consistent estimator. Next,

$$
\begin{aligned}
\bar{E}\{\hat{\boldsymbol{\mu}}\}= & \lim _{\substack{n \rightarrow \infty \\
T \rightarrow \infty}} E\{\hat{\boldsymbol{\mu}}\} \\
= & \boldsymbol{\mu}+\left(\lim _{\substack{n \rightarrow \infty \\
T \rightarrow \infty}} \frac{1}{n T}\left[\frac{1}{n} \mathbf{G}^{t} \hat{\mathbf{H}}^{*} \mathbf{G}\right]^{-1}\right)\left(\sum_{j=1}^{T} \mathbf{G}^{t} \hat{\mathbf{H}}^{*} \mathbf{Z}_{j}\right. \\
& \left.\times\left(\boldsymbol{\theta}-\lim _{\substack{n \rightarrow \infty \\
T \rightarrow \infty}}\{\hat{\boldsymbol{\theta}}\}\right)+\lim _{\substack{n \rightarrow \infty \\
T \rightarrow \infty}} \sum_{j=1}^{T} \mathbf{G}^{t} \hat{\mathbf{H}}^{*} \hat{\mathbf{B}}_{*} E\left\{\boldsymbol{\varepsilon}_{j}\right\}\right) \\
= & \boldsymbol{\mu}+\left(\lim _{\substack{n \rightarrow \infty \\
T \rightarrow \infty}} \frac{1}{n T}\left[\frac{1}{n} \mathbf{K}_{1}\right]^{-1}\right)\left(\sum_{j=1}^{T} \mathbf{G}^{t} \hat{\mathbf{H}}^{*} \mathbf{Z}_{j}(\boldsymbol{\theta}-\boldsymbol{\theta})\right. \\
& \left.+\lim _{\substack{n \rightarrow \infty \\
T \rightarrow \infty}} \sum_{\substack{j=1 \\
\text { G }}}^{t} \hat{\mathbf{H}}^{*} \hat{\mathbf{B}}_{*} \times \mathbf{0}\right) \\
= & \boldsymbol{\mu}+\left(\lim _{\substack{n \rightarrow \infty \\
T \rightarrow \infty}} \frac{1}{n T}\left[\overline{\mathbf{K}}_{1}\right]^{-1}\right) \times(\mathbf{0}+\mathbf{0})=\boldsymbol{\mu},
\end{aligned}
$$

where $\mathbf{K}_{1}$ and $\overline{\mathbf{K}}_{1}$ are constant nonsingular matrices. We have asy. $\operatorname{var}\{\hat{\boldsymbol{\mu}}\}=$ asy.var $\left\{\left[T \mathbf{G}^{t} \hat{\mathbf{H}}^{*} \mathbf{G}\right]^{-1} \sum_{j=1}^{T} \mathbf{G}^{t} \hat{\mathbf{H}}^{*} \mathbf{Z}_{j} \hat{\boldsymbol{\theta}}\right\}$ + asy.var $\left\{\left[T \mathbf{G}^{t} \hat{\mathbf{H}}^{*} \mathbf{G}\right]^{-1} \sum_{j=1}^{T} \mathbf{G}^{t} \hat{\mathbf{H}}^{*} \hat{\mathbf{B}}_{*} \boldsymbol{\varepsilon}_{j}\right\}$,

asy. $\operatorname{var}\left\{\left[T \mathbf{G}^{t} \hat{\mathbf{H}}^{*} \mathbf{G}\right]^{-1} \sum_{j=1}^{T} \mathbf{G}^{t} \hat{\mathbf{H}}^{*} \mathbf{Z}_{j} \hat{\boldsymbol{\theta}}\right\}=\left[T \mathbf{G}^{t} \hat{\mathbf{H}}^{*} \mathbf{G}\right]^{-1}$ $\times\left[\sum_{j=1}^{T} \mathbf{G}^{t} \hat{\mathbf{H}}^{*} \mathbf{Z}_{j}\right.$ asy.var $\left.\{\hat{\boldsymbol{\theta}}\} \mathbf{Z}_{j}^{t} \hat{\mathbf{H}}^{*} \mathbf{G}\right]\left[T \mathbf{G}^{t} \hat{\mathbf{H}}^{*} \mathbf{G}\right]^{-1}$, asy. $\operatorname{var}\left\{\left[T \mathbf{G}^{t} \hat{\mathbf{H}}^{*} \mathbf{G}\right]^{-1} \sum_{j=1}^{T} \mathbf{G}^{t} \hat{\mathbf{H}}^{*} \hat{\mathbf{B}}_{*} \boldsymbol{\varepsilon}_{j}\right\}=\left[T \mathbf{G}^{t} \hat{\mathbf{H}}^{*} \mathbf{G}\right]^{-1}$ $\times\left[T \mathbf{G}^{t} \hat{\mathbf{H}}^{*} \hat{\mathbf{B}}_{*}\left(\boldsymbol{\Sigma} \otimes \mathbf{I}_{n}\right) \hat{\mathbf{B}}_{*}^{t} \hat{\mathbf{H}}^{*} \mathbf{G}\right]\left[T \mathbf{G}^{t} \hat{\mathbf{H}}^{*} \mathbf{G}\right]^{-1}$ $=\left[\mathbf{G}^{t} \hat{\mathbf{H}}^{*} \mathbf{G}\right]^{-1}\left[\mathbf{G}^{t} \hat{\mathbf{H}}^{*} \hat{\mathbf{B}}_{*}\left(\boldsymbol{\Sigma} \otimes \mathbf{I}_{n}\right) \hat{\mathbf{B}}_{*}^{t} \hat{\mathbf{H}}^{*} \mathbf{G}\right]$ $\times\left[T \mathbf{G}^{t} \hat{\mathbf{H}}^{*} \mathbf{G}\right]^{-1}$.

$$
\begin{aligned}
\lim _{\substack{n \rightarrow \infty \\
T \rightarrow \infty}} \text { asy. var }\left\{\left[T \mathbf{G}^{t} \hat{\mathbf{H}}^{*} \mathbf{G}\right]^{-1} \sum_{j=1}^{T} \mathbf{G}^{t} \hat{\mathbf{H}}^{*} \mathbf{Z}_{j} \hat{\boldsymbol{\theta}}\right\} \\
=\lim _{\substack{n \rightarrow \infty \\
T \rightarrow \infty}} \frac{1}{n T}\left[\frac{1}{n} \mathbf{G}^{t} \hat{\mathbf{H}}^{*} \mathbf{G}\right]^{-1}\left[\sum_{j=1}^{T} \mathbf{G}^{t} \hat{\mathbf{H}}^{*} \mathbf{Z}_{j}\left(\lim _{\substack{n \rightarrow \infty \\
T \rightarrow \infty}} \operatorname{asy} \cdot \operatorname{var}\{\hat{\boldsymbol{\theta}}\}\right)\right. \\
\left.\quad \times \mathbf{Z}_{j}^{t} \hat{\mathbf{H}}^{*} \mathbf{G}\right]_{\substack{n \rightarrow \infty \\
T \rightarrow \infty}} \frac{1}{n T}\left[\frac{1}{n} \mathbf{G}^{t} \hat{\mathbf{H}}^{*} \mathbf{G}\right]^{-1} \\
=\lim _{\substack{n \rightarrow \infty \\
T \rightarrow \infty}} \frac{1}{n T}\left[\overline{\mathbf{K}}_{1}\right]^{-1}\left[\sum_{j=1}^{T} \mathbf{G}^{t} \hat{\mathbf{H}}^{*} \mathbf{Z}_{j} \times \mathbf{0} \times \mathbf{Z}_{j}^{t} \hat{\mathbf{H}}^{*} \mathbf{G}\right] \\
\quad \times \lim _{\substack{n \rightarrow \infty \\
T \rightarrow \infty}} \frac{1}{n T}\left[\overline{\mathbf{K}}_{1}\right]^{-1} \\
=\mathbf{0} \times \mathbf{0} \times \mathbf{0}=\mathbf{0} .
\end{aligned}
$$

$\lim _{\substack{n \rightarrow \infty \\ T \rightarrow \infty}} \operatorname{asy} . \operatorname{var}\left\{\left[T \mathbf{G}^{t} \hat{\mathbf{H}}^{*} \mathbf{G}\right]^{-1} \sum_{j=1}^{T} \mathbf{G}^{t} \hat{\mathbf{H}}^{*} \hat{\mathbf{B}}_{*} \boldsymbol{\varepsilon}_{j}\right\}$ $=\left[\mathbf{G}^{t} \hat{\mathbf{H}}^{*} \mathbf{G}\right]^{-1}\left[\mathbf{G}^{t} \hat{\mathbf{H}}^{*} \hat{\mathbf{B}}_{*}\left\{\lim _{\substack{n \rightarrow \infty \\ T \rightarrow \infty}} \frac{1}{n T}\left(\mathbf{\Sigma} \otimes \mathbf{I}_{n}\right)\right\} \hat{\mathbf{B}}_{*}^{t} \hat{\mathbf{H}}^{*} \mathbf{G}\right]$ $\times\left[\lim _{\substack{n \rightarrow \infty \\ T \rightarrow \infty}} \frac{1}{n} \mathbf{G}^{t} \hat{\mathbf{H}}^{*} \mathbf{G}\right]^{-1}$ $=\left[\mathbf{K}_{1}\right]^{-1}\left[\mathbf{G}^{t} \hat{\mathbf{H}}^{*} \hat{\mathbf{B}}_{*} \times \mathbf{0} \times \hat{\mathbf{B}}_{*}^{t} \hat{\mathbf{H}}^{*} \mathbf{G}\right]\left[\lim _{\substack{n \rightarrow \infty \\ T \rightarrow \infty}} \overline{\mathbf{K}}_{1}\right]^{-1}$ $=\mathbf{K}_{1}^{-1} \times \mathbf{0} \times\left[\overline{\mathbf{K}}_{1}\right]^{-1}=\mathbf{0}$.

Consequently, $\lim _{\substack{n \rightarrow \infty \\ T \rightarrow \infty}} \operatorname{asy} . \operatorname{var}\{\hat{\boldsymbol{\mu}}\}=\mathbf{0}$.

This shows that $\hat{\boldsymbol{\mu}}$ is asymptotically unbiased estimator. If $n \rightarrow \infty$ or $T \rightarrow \infty$ or both of $n \rightarrow \infty$ and $T \rightarrow \infty$, then asy. $\operatorname{var}\{\hat{\boldsymbol{\mu}}\} \rightarrow \mathbf{0}$. Therefore, $\hat{\boldsymbol{\mu}}$ is a consistent estimator. Now, 


$$
\begin{aligned}
\bar{E}\left\{\hat{\boldsymbol{\gamma}}_{j}\right\}= & \lim _{\substack{n \rightarrow \infty \\
T \rightarrow \infty}} E\left\{\hat{\boldsymbol{\gamma}}_{j}\right\} \\
= & \left(\boldsymbol{\mu}-\lim _{\substack{n \rightarrow \infty \\
T \rightarrow \infty}} E\{\hat{\boldsymbol{\mu}}\}\right)+\left[\mathbf{G}^{t} \hat{\mathbf{H}}^{*} \mathbf{G}\right]^{-1} \mathbf{G}^{t} \hat{\mathbf{H}}^{*} \mathbf{Z}_{j} \\
& \times\left(\boldsymbol{\theta}-\lim _{\substack{n \rightarrow \infty \\
T \rightarrow \infty}} E\{\hat{\boldsymbol{\theta}}\}\right)+\boldsymbol{\gamma}_{j}+\lim _{\substack{n \rightarrow \infty \\
T \rightarrow \infty}} \frac{1}{n}\left[\frac{1}{n} \mathbf{G}^{t} \hat{\mathbf{H}}^{*} \mathbf{G}\right]^{-1} \\
& \times \mathbf{G}^{t} \hat{\mathbf{H}}^{*} \hat{\mathbf{B}}_{*} E\left\{\boldsymbol{\varepsilon}_{j}\right\}
\end{aligned}
$$

$$
\begin{aligned}
\bar{E}\left\{\hat{\boldsymbol{\gamma}}_{j}\right\}= & (\boldsymbol{\mu}-\boldsymbol{\mu})+\left[\mathbf{K}_{1}\right]^{-1} \mathbf{G}^{t} \hat{\mathbf{H}}^{*} \mathbf{Z}_{j}(\boldsymbol{\theta}-\boldsymbol{\theta})+\boldsymbol{\gamma}_{j} \\
& +\lim _{\substack{n \rightarrow \infty \\
T \rightarrow \infty}} \frac{1}{n}\left[\overline{\mathbf{K}}_{1}\right]^{-1} \mathbf{G}^{t} \hat{\mathbf{H}}^{*} \hat{\mathbf{B}}_{*} \times \mathbf{0} \\
= & \boldsymbol{\gamma}_{j} .
\end{aligned}
$$

asy. $\operatorname{var}\left\{\hat{\boldsymbol{\gamma}}_{j}\right\}=$ asy. $\operatorname{var}\left\{(\boldsymbol{\mu}-\hat{\boldsymbol{\mu}})+\left[\mathbf{G}^{t} \hat{\mathbf{H}}^{*} \mathbf{G}\right]^{-1}\right.$

$$
\begin{aligned}
& \times \mathbf{G}^{t} \hat{\mathbf{H}}^{*} \mathbf{Z}_{j}(\boldsymbol{\theta}-\hat{\boldsymbol{\theta}})+\boldsymbol{\gamma}_{j} \\
& \left.+\left[\mathbf{G}^{t} \hat{\mathbf{H}}^{*} \mathbf{G}\right]^{-1} \mathbf{G}^{t} \hat{\mathbf{H}}^{*} \hat{\mathbf{B}}_{*} \boldsymbol{\varepsilon}_{j}\right\} \\
= & \operatorname{asy} \cdot \operatorname{var}\{\hat{\boldsymbol{\mu}}\} \\
& +\operatorname{asy} \cdot \operatorname{var}\left\{\left[\mathbf{G}^{t} \hat{\mathbf{H}}^{*} \mathbf{G}\right]^{-1} \mathbf{G}^{t} \hat{\mathbf{H}}^{*} \mathbf{Z}_{j} \hat{\boldsymbol{\theta}}\right\} \\
& +\operatorname{asy} \cdot \operatorname{var}\left\{\left[\mathbf{G}^{t} \hat{\mathbf{H}}^{*} \mathbf{G}\right]^{-1} \mathbf{G}^{t} \hat{\mathbf{H}}^{*} \hat{\mathbf{B}}_{*} \boldsymbol{\varepsilon}_{j}\right\} .
\end{aligned}
$$

$$
\begin{aligned}
\operatorname{asy.var} & \left\{\left[\mathbf{G}^{t} \hat{\mathbf{H}}^{*} \mathbf{G}\right]^{-1} \mathbf{G}^{t} \hat{\mathbf{H}}^{*} \mathbf{Z}_{j} \hat{\boldsymbol{\theta}}\right\} \\
= & {\left[\mathbf{G}^{t} \hat{\mathbf{H}}^{*} \mathbf{G}\right]^{-1} \mathbf{G}^{t} \hat{\mathbf{H}}^{*} \mathbf{Z}_{j} \text { asy.var }\{\hat{\boldsymbol{\theta}}\} } \\
& \times \mathbf{Z}_{j}^{t} \hat{\mathbf{H}}^{*} \mathbf{G}\left[\mathbf{G}^{t} \hat{\mathbf{H}}^{*} \mathbf{G}\right]^{-1},
\end{aligned}
$$$$
\text { asy.var }\left\{\left[\mathbf{G}^{t} \hat{\mathbf{H}}^{*} \mathbf{G}\right]^{-1} \mathbf{G}^{t} \hat{\mathbf{H}}^{*} \hat{\mathbf{B}}_{*} \boldsymbol{\varepsilon}_{j}\right\}
$$$$
\begin{aligned}
= & {\left[\mathbf{G}^{t} \hat{\mathbf{H}}^{*} \mathbf{G}\right]^{-1} \mathbf{G}^{t} \hat{\mathbf{H}}^{*} \hat{\mathbf{B}}_{*}\left(\boldsymbol{\Sigma} \otimes \mathbf{I}_{n}\right) } \\
& \times \hat{\mathbf{B}}_{*}^{t} \hat{\mathbf{H}}^{*} \mathbf{G}\left[\mathbf{G}^{t} \hat{\mathbf{H}}^{*} \mathbf{G}\right]^{-1} .
\end{aligned}
$$

$\lim _{\substack{n \rightarrow \infty \\ T \rightarrow \infty}} \operatorname{asy} \cdot \operatorname{var}\left\{\left[\mathbf{G}^{t} \hat{\mathbf{H}}^{*} \mathbf{G}\right]^{-1} \mathbf{G}^{t} \hat{\mathbf{H}}^{*} \mathbf{Z}_{j} \hat{\boldsymbol{\theta}}\right\}$

$$
\begin{aligned}
= & {\left[\mathbf{G}^{t} \hat{\mathbf{H}}^{*} \mathbf{G}\right]^{-1} \mathbf{G}^{t} \hat{\mathbf{H}}^{*} \mathbf{Z}_{j}\left(\lim _{\substack{n \rightarrow \infty \\
T \rightarrow \infty}} \operatorname{asy} \cdot \operatorname{var}\{\hat{\boldsymbol{\theta}}\}\right) } \\
& \times \mathbf{Z}_{j}^{t} \hat{\mathbf{H}}^{*} \mathbf{G}\left[\mathbf{G}^{t} \hat{\mathbf{H}}^{*} \mathbf{G}\right]^{-1} \\
= & {\left[\mathbf{K}_{1}\right]^{-1} \mathbf{G}^{t} \hat{\mathbf{H}}^{*} \mathbf{Z}_{j} \times \mathbf{0} \times \mathbf{Z}_{j}^{t} \hat{\mathbf{H}}^{*} \mathbf{G}\left[\mathbf{K}_{1}\right]^{-1} } \\
= & \mathbf{0}
\end{aligned}
$$

$$
\begin{aligned}
& \lim _{\substack{n \rightarrow \infty \\
T \rightarrow \infty}} \operatorname{asy} . \operatorname{var}\left\{\left[\mathbf{G}^{t} \hat{\mathbf{H}}^{*} \mathbf{G}\right]^{-1} \mathbf{G}^{t} \hat{\mathbf{H}}^{*} \hat{\mathbf{B}}_{*} \boldsymbol{\varepsilon}_{j}\right\} \\
& =\left[\mathbf{G}^{t} \hat{\mathbf{H}}^{*} \mathbf{G}\right]^{-1} \mathbf{G}^{t} \hat{\mathbf{H}}^{*} \hat{\mathbf{B}}_{*}\left\{\lim _{\substack{n \rightarrow \infty \\
T \rightarrow \infty}} \frac{1}{n T}\left(\boldsymbol{\Sigma} \otimes \mathbf{I}_{n}\right)\right\} \\
& \times \hat{\mathbf{B}}_{*}^{t} \hat{\mathbf{H}}^{*} \mathbf{G}\left[\lim _{\substack{n \rightarrow \infty \\
T \rightarrow \infty}} \frac{1}{T} \frac{1}{n} \mathbf{G}^{t} \hat{\mathbf{H}}^{*} \mathbf{G}\right]^{-1} \\
& =\left[\mathbf{K}_{1}\right]^{-1} \mathbf{G}^{t} \hat{\mathbf{H}}^{*} \hat{\mathbf{B}}_{*} \times \mathbf{0} \times \hat{\mathbf{B}}_{*}^{t} \hat{\mathbf{H}}^{*} \mathbf{G}\left[\lim _{\substack{n \rightarrow \infty \\
T \rightarrow \infty}} \frac{1}{T} \overline{\mathbf{K}}_{1}\right]^{-1} \\
& =\mathbf{0} \times[\mathbf{0}]^{-1}=\infty \text { (infinit), }
\end{aligned}
$$

therefore, convergenity be satisfied only if $n \rightarrow \infty$, namely

$$
\begin{aligned}
& \lim _{n \rightarrow \infty} \text { asy. var }\left\{\left[\mathbf{G}^{t} \hat{\mathbf{H}}^{*} \mathbf{G}\right]^{-1} \mathbf{G}^{t} \hat{\mathbf{H}}^{*} \hat{\mathbf{B}}_{*} \boldsymbol{\varepsilon}_{j}\right\} \\
&=\left[\mathbf{G}^{t} \hat{\mathbf{H}}^{*} \mathbf{G}\right]^{-1} \mathbf{G}^{t} \hat{\mathbf{H}}^{*} \hat{\mathbf{B}}_{*}\left\{\lim _{n \rightarrow \infty} \frac{1}{n}\left(\mathbf{\Sigma} \otimes \mathbf{I}_{n}\right)\right\} \\
& \quad \times \hat{\mathbf{B}}_{*}^{t} \hat{\mathbf{H}}^{*} \mathbf{G}\left[\lim _{n \rightarrow \infty} \frac{1}{n} \mathbf{G}^{t} \hat{\mathbf{H}}^{*} \mathbf{G}\right]^{-1} \\
&=\left[\mathbf{K}_{1}\right]^{-1} \mathbf{G}^{t} \hat{\mathbf{H}}^{*} \hat{\mathbf{B}}_{*} \times \mathbf{0} \times \hat{\mathbf{B}}_{*}^{t} \hat{\mathbf{H}}^{*} \mathbf{G}\left[\lim _{n \rightarrow \infty} \overline{\mathbf{K}}_{1}\right]^{-1} \\
&= \mathbf{K}_{1}^{-1} \times \mathbf{0} \times\left[\overline{\mathbf{K}}_{1}\right]^{-1}=\mathbf{0} .
\end{aligned}
$$

Consequently, $\lim _{n \rightarrow \infty} \operatorname{asy} \cdot \operatorname{var}\left\{\hat{\boldsymbol{\gamma}}_{j}\right\}=\mathbf{0}$.

This shows that $\hat{\gamma}_{j}$ is asymptotically unbiased estimator. If $n \rightarrow \infty$, then asy.var $\left\{\hat{\boldsymbol{\gamma}}_{j}\right\} \rightarrow \mathbf{0}$. Therefore, $\hat{\gamma}_{j}$ is a consistent estimator.

\section{Illustration}

Suppose there are three endogenous variables $y_{1}, y_{2}, y_{3}$ and six exogenous variables $x_{11}, x_{12}, x_{21}, x_{22}, x_{31}, x_{32}$ observed for two time periods and the number of observation being 10 locations. We use illustration of data, locations, and row-standardized spatial weight matrix as it was presented in [2]. The equation models are as follows:

$$
\begin{aligned}
y_{1 i j}= & \mu_{1}+\alpha_{11} x_{11 i j}+\alpha_{12} x_{12 i j}+\rho_{1} \mathbf{w}_{i}^{t} \mathbf{y}_{1 j}+\beta_{12} y_{2 i j} \\
& +\beta_{13} y_{3 i j}+\gamma_{1 j}+u_{1 i j} \\
y_{2 i j}= & \mu_{2}+\alpha_{21} x_{21 i j}+\alpha_{22} x_{22 i j}+\rho_{2} \mathbf{w}_{i}^{t} \mathbf{y}_{2 j}+\beta_{21} y_{1 i j} \\
& +\beta_{23} y_{3 i j}+\gamma_{2 j}+u_{2 i j} \\
y_{3 i j}= & \mu_{3}+\alpha_{31} x_{31 i j}+\alpha_{32} x_{32 i j}+\rho_{3} \mathbf{w}_{i}^{t} \mathbf{y}_{3 j}+\beta_{31} y_{1 i j} \\
& +\beta_{32} y_{2 i j}+\gamma_{3 j}+u_{3 i j},
\end{aligned}
$$




$$
\begin{aligned}
& u_{1 i j}=\lambda_{1} \mathbf{w}_{i}^{t} \mathbf{u}_{1 j}+\varepsilon_{1 i j}, \quad \varepsilon_{1 i j} \square N\left(0, \sigma_{1}^{2}\right), \\
& u_{2 i j}=\lambda_{2} \mathbf{w}_{i}^{t} \mathbf{u}_{2 j}+\varepsilon_{2 i j}, \quad \varepsilon_{2 i j} \square N\left(0, \sigma_{2}^{2}\right), \\
& u_{3 i j}=\lambda_{3} \mathbf{w}_{i}^{t} \mathbf{u}_{3 j}+\varepsilon_{3 i j}, \quad \varepsilon_{3 i j} \square N\left(0, \sigma_{3}^{2}\right),
\end{aligned}
$$

where

$$
\begin{aligned}
\mathbf{W} & =\left[\begin{array}{ccccc}
w_{11} & w_{12} & w_{13} & \cdots & w_{1,10} \\
w_{21} & w_{22} & w_{23} & \cdots & w_{2,10} \\
w_{31} & w_{32} & w_{33} & \cdots & w_{3,10} \\
\vdots & \vdots & \vdots & \ddots & \vdots \\
w_{10,1} & w_{10,2} & w_{10,3} & \cdots & w_{10,10}
\end{array}\right]=\left[\begin{array}{c}
\mathbf{w}_{1}^{t} \\
\mathbf{w}_{2}^{t} \\
\mathbf{w}_{3}^{t} \\
\vdots \\
\mathbf{w}_{10}^{t}
\end{array}\right] \\
& =\left[\mathbf{w}_{i}^{t}\right], \quad i=1,2,3, \cdots, 10,
\end{aligned}
$$

The formulation of Moran Index is as follows:

$$
I_{h j}=\frac{\sum_{i=1}^{10} \sum_{i^{*}=1}^{10} w_{i i^{*}}\left(y_{h i j}-\bar{y}_{h j}\right)\left(y_{h i^{*} j}-\bar{y}_{h j}\right)}{\sum_{i=1}^{10}\left(y_{h i j}-\bar{y}_{h j}\right)^{2}}=\frac{\mathbf{y}_{h j}^{*} \mathbf{W}_{h j}^{*}}{\mathbf{y}_{h j}^{* t} \mathbf{y}_{h j}^{*}},
$$

for $h=1,2,3, j=1,2$,

where $\bar{y}_{h j}=\frac{1}{10} \sum_{i=1}^{10} y_{h i j}$ and $\mathbf{y}_{h j}^{*}=\mathbf{y}_{h j}-\bar{y}_{h j} \mathbf{1}$.

If there is at least one $I_{h j}>E(I)$, then we conclude that there is a spatial influence for the equation models.

$$
\begin{aligned}
& \bar{y}_{11}=15.80 ; \quad \bar{y}_{21}=28.30 ; \quad \bar{y}_{31}=24.90 ; \\
& \bar{y}_{22}=28.40 ; \quad \bar{y}_{32}=25.90 ; \\
& I_{11}=-0.2442 ; I_{21}=0.0539 ; I_{31}=0.4586 ; \\
& I_{12}=-0.2317 ; I_{22}=-0.0878 ; I_{32}=-0.1078 ;
\end{aligned}
$$

$$
\text { and } E\left(I_{h j}\right)=E(I)=\frac{-1}{n-1}=\frac{-1}{10-1}=-0.1111 \text {. }
$$

Based on the above result, by means of R Program version 3.6.1, we obtain that there is a spatial influence for the equation models.

We then continue to estimate parameters by means of FGLS-3SLS. For the first-stage, we estimate all the endogenous expalanatory variables in the system in every time period and the results are presented in Table 1.

For the second-stage we estimate $\boldsymbol{\Sigma}_{\# \text {. }}$ But, we first estimate both of spatial autoregressive and spatial autocorrelation by means of equation (17). By $\mathbf{W}$ matrix, we have the acceptable spatial autoregressive and spatial autocorrelation parameters are $-1.6242<\rho_{h}\left(\lambda_{h}\right)<1$. By method of forming sequence both of $\rho_{h}$ and $\lambda_{h}$ with increasing 0.01 we obtain

1. $\rho_{h}=\operatorname{seq}(-1.6142,0.99,0.01)$ and

$$
\lambda_{h}=\operatorname{seq}(-1.6142,0.99,0.01) \text {. }
$$

\begin{tabular}{|c|c|c|c|c|}
\hline \multirow{2}{*}{ Time } & \multirow{2}{*}{$\begin{array}{l}\text { Loca- } \\
\text { tion }\end{array}$} & \multicolumn{3}{|c|}{$\begin{array}{c}\text { Endogenous explanatory } \\
\text { variables }\end{array}$} \\
\hline & & $\begin{array}{c}y_{1-}^{-} \\
\text {estimate }\end{array}$ & $\begin{array}{c}y_{2-} \\
\text { estimate }\end{array}$ & $\begin{array}{c}y_{3^{-}} \\
\text {estimate }\end{array}$ \\
\hline \multirow[t]{10}{*}{1} & 1 & 16.5625 & 26.5828 & 21.7290 \\
\hline & 2 & 15.0373 & 28.5890 & 25.1588 \\
\hline & 3 & 16.1904 & 27.6672 & 20.7955 \\
\hline & 4 & 12.3775 & 26.0621 & 23.9145 \\
\hline & 5 & 16.1804 & 28.3403 & 22.0819 \\
\hline & 6 & 17.2918 & 26.9959 & 24.8593 \\
\hline & 7 & 18.7007 & 29.1060 & 26.5129 \\
\hline & 8 & 12.3543 & 31.5231 & 28.7592 \\
\hline & 9 & 16.4805 & 31.4345 & 30.8012 \\
\hline & 10 & 16.8246 & 26.6991 & 24.3877 \\
\hline \multirow[t]{10}{*}{2} & 1 & 15.5100 & 25.9073 & 23.1069 \\
\hline & 2 & 17.3247 & 27.0314 & 24.7638 \\
\hline & 3 & 15.8433 & 26.3597 & 22.8089 \\
\hline & 4 & 13.3019 & 25.4562 & 21.0773 \\
\hline & 5 & 17.3259 & 29.7492 & 27.8872 \\
\hline & 6 & 18.1930 & 30.2621 & 28.3106 \\
\hline & 7 & 18.0785 & 31.1379 & 29.8797 \\
\hline & 8 & 14.7653 & 32.0924 & 30.1569 \\
\hline & 9 & 14.9671 & 29.3371 & 27.3870 \\
\hline & 10 & 18.6902 & 26.6667 & 23.6217 \\
\hline
\end{tabular}

2. For every $\mathbf{y}_{h j}$ and $\mathbf{a}_{h j}, h=1,2,3$, we insert combination of all possible values of $\rho_{h}$ and $\lambda_{h}$ to (17). Because $\mathbf{a}_{h j}$ is unknown, we use the estimate $\hat{\mathbf{a}}_{h j}$, where $\hat{\mathbf{a}}_{h j}=\mathbf{1}\left(\hat{\mu}_{h}+\hat{\gamma}_{h j}\right)+\mathbf{Z}_{h j} \hat{\boldsymbol{\theta}}_{h}$, with $\mathbf{Z}_{h j}=\left[\begin{array}{lll}\mathbf{X}_{h j} & \vdots & \hat{\mathbf{Y}}_{-h j}\end{array}\right]$.

3. We obtain $\hat{\rho}_{1}=-1.6142, \quad \hat{\lambda}_{1}=0.9258$, $\hat{\rho}_{2}=-1.6142, \quad \hat{\lambda}_{2}=-0.2242, \quad \hat{\rho}_{3}=-1.5742$, and $\hat{\lambda}_{3}=0.4658$ those give the largest $\ln L_{1}^{c o n}, \ln L_{2}^{c o n}$ and $\ln L_{3}^{c o n}$, respectively.

Table 1 Estimated values for endogenous explanatory variables

By sequences of $\rho_{h}$ and $\lambda_{h}$ with increasing 0.01 , we can also make graphs among the values of rho, lambda, and the values of concentrated loglikelihood as presented in Fig.1. 


\section{Graph of function InLcon1 of rho1 and lambda1}

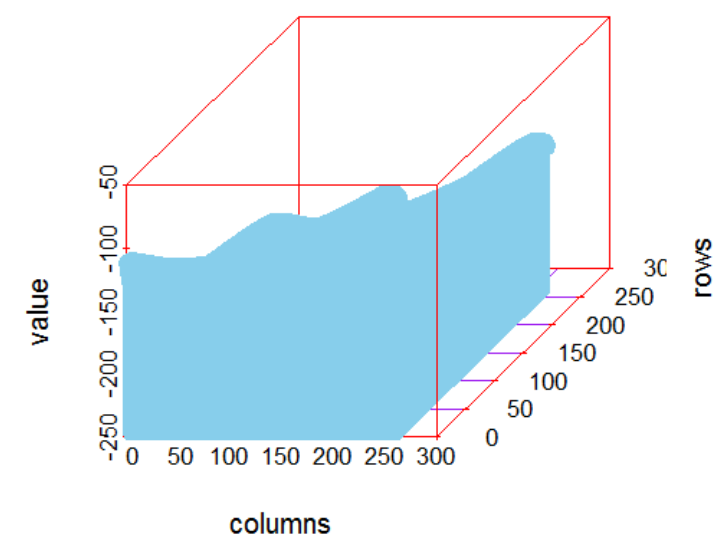

Graph of function InLcon2 of rho2 and lambda2

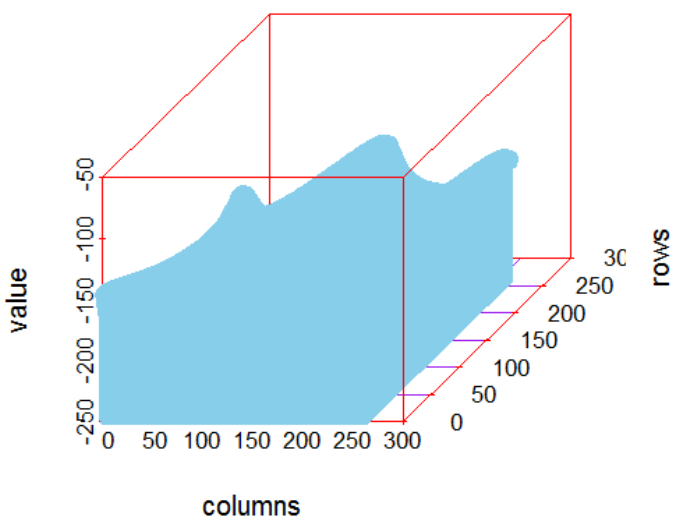

Graph of function InLcon3 of rho3 and lambda3

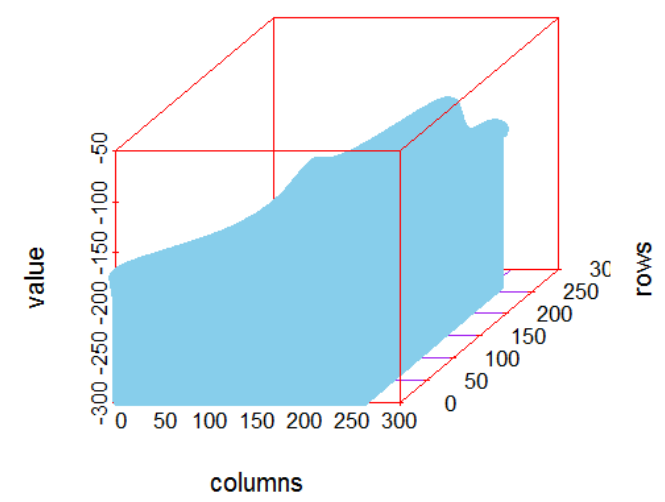

Fig.1: Graphs of function of rho and lambda
As we need to know in Fig. 1, rows are the points of rho that its values are seq(-1.6142, 0.99, 0.01), columns are the points of lambda that its values are seq $(-1.6142,0.99,0.01)$, and value is the value of lnLcon. There are 261 points both of rho and lambda. Its points are 1,2,3, untill 261.

Table 2 Estimate values for residual errors

\begin{tabular}{|c|c|c|c|c|}
\hline \multirow[b]{2}{*}{ Time } & \multirow{2}{*}{$\begin{array}{l}\text { Loca- } \\
\text { tion }\end{array}$} & \multicolumn{3}{|c|}{ Residual errors } \\
\hline & & $\begin{array}{c}u_{1^{-}} \\
\text {estimate }\end{array}$ & $\begin{array}{c}u_{2-} \\
\text { estimate }\end{array}$ & $\begin{array}{c}u_{3^{-}} \\
\text {estimate }\end{array}$ \\
\hline \multirow[t]{10}{*}{1} & 1 & 530.9617 & 3.6731 & -20.1653 \\
\hline & 2 & 634.4891 & 123.6771 & -30.2136 \\
\hline & 3 & 708.4265 & -83.8497 & 20.1640 \\
\hline & 4 & 821.6256 & 69.4616 & 11.7260 \\
\hline & 5 & 677.8279 & -63.3284 & 14.2880 \\
\hline & 6 & 612.9724 & 93.3877 & 78.6841 \\
\hline & 7 & 612.4407 & 151.2321 & 62.3801 \\
\hline & 8 & 570.8471 & 245.4605 & -133.6357 \\
\hline & 9 & 609.1129 & 280.6660 & 10.3691 \\
\hline & 10 & 560.6974 & 109.3295 & 57.4756 \\
\hline \multirow[t]{10}{*}{2} & 1 & $1,021.2697$ & -122.8737 & 58.6738 \\
\hline & 2 & $1,005.5465$ & -99.2300 & 60.9696 \\
\hline & 3 & 973.8582 & -236.6423 & 73.0186 \\
\hline & 4 & 945.0748 & -280.0563 & 73.3787 \\
\hline & 5 & 748.1006 & 33.4929 & -10.1951 \\
\hline & 6 & 846.8442 & -25.4341 & 16.2969 \\
\hline & 7 & 828.7046 & 73.9246 & -26.9565 \\
\hline & 8 & 801.7122 & 79.0934 & -50.7021 \\
\hline & 9 & 927.9174 & -78.3885 & -50.9108 \\
\hline & 10 & 932.3442 & -215.1956 & 104.0915 \\
\hline
\end{tabular}

From (18) to (20), we obtain

$\hat{\mu}_{1}=59.8488 ; \quad \hat{\mu}_{2}=35.7021 ; \quad \hat{\mu}_{3}=30.2172 ;$

$\hat{\gamma}_{11}=-4.7589 ; \hat{\gamma}_{12}=4.7589$;

$\hat{\gamma}_{21}=3.0530 ; \hat{\gamma}_{22}=-3.0530$;

$\hat{\gamma}_{31}=1.2893 ; \hat{\gamma}_{32}=-1.2893$.

$\hat{\boldsymbol{\theta}}_{1}=\left[\begin{array}{l}\hat{\boldsymbol{\alpha}}_{1} \\ \cdots \\ \hat{\boldsymbol{\beta}}_{-1}\end{array}\right]=\left[\begin{array}{l}\hat{\alpha}_{11} \\ \hat{\alpha}_{12} \\ \hat{\beta}_{12} \\ \hat{\beta}_{13}\end{array}\right]=\left[\begin{array}{r}7.5482 \\ -36.8974 \\ 34.8812 \\ 3.1676\end{array}\right] ;$ 


$$
\begin{gathered}
\hat{\boldsymbol{\theta}}_{2}=\left[\begin{array}{l}
\hat{\boldsymbol{\alpha}}_{2} \\
\ldots \\
\hat{\boldsymbol{\beta}}_{-2}
\end{array}\right]=\left[\begin{array}{l}
\hat{\alpha}_{21} \\
\hat{\alpha}_{22} \\
\hat{\beta}_{21} \\
\hat{\beta}_{23}
\end{array}\right]=\left[\begin{array}{r}
7.9886 \\
10.6995 \\
\cdots \\
6.2486 \\
-44.5616
\end{array}\right] ; \\
\hat{\boldsymbol{\theta}}_{3}=\left[\begin{array}{l}
\hat{\boldsymbol{\alpha}}_{3} \\
\cdots \\
\hat{\boldsymbol{\beta}}_{-3}
\end{array}\right]=\left[\begin{array}{l}
\hat{\alpha}_{31} \\
\hat{\alpha}_{32} \\
\hat{\beta}_{31} \\
\hat{\beta}_{32}
\end{array}\right]=\left[\begin{array}{r}
8.2808 \\
-7.9064 \\
-23.8113 \\
16.0997
\end{array}\right] ;
\end{gathered}
$$

Next, from (21) we obtain the estimate values for residual errors being presented in Tabel 2. We then use the estimate values for residual errors (in Table 2) to find $\hat{\boldsymbol{\Sigma}}$ as follow:

$$
\hat{\boldsymbol{\Sigma}}=\left[\begin{array}{rrr}
16,407.7430 & -13,327.4840 & 3,586.3450 \\
-13,327.4840 & 55,037.3740 & -6,806.3970 \\
3,586.3450 & -6,806.3970 & 8,201.1720
\end{array}\right] \text {, }
$$

and we obtain

$$
\begin{aligned}
& \hat{\boldsymbol{\Sigma}}_{\#}=\left[\begin{array}{rr}
95,015,762,019 & 112,938,200,311 \\
112,938,200,311 & 134,249,432,552 \\
95,961,464,592 & 114,062,507,032 \\
\vdots & \vdots \\
3,116,649,203 & 3,704,593,381
\end{array}\right. \\
& 95,961,464,592 \quad \cdots \quad 3,116,649,203 \\
& \begin{array}{rll}
114,062,507,032 & \cdots & 3,704,593,381
\end{array} \\
& 96,916,769,558 \quad \cdots \quad 3,147,439,542 \text {, } \\
& 3,147,439,542 \quad \cdots \quad 1,100,342,293]
\end{aligned}
$$

where $\hat{\boldsymbol{\Sigma}}_{\#}$ is the estimator of covariance matrix.

For the last-stage, we estimate the parameters of equation models (25). From (22) to (24), we obtain

$$
\hat{\boldsymbol{\theta}}=\left[\begin{array}{l}
\alpha_{11} \\
\alpha_{12} \\
\alpha_{21} \\
\alpha_{22} \\
\alpha_{31} \\
\alpha_{32} \\
\beta_{12} \\
\beta_{13} \\
\beta_{21} \\
\beta_{23} \\
\beta_{31} \\
\beta_{32}
\end{array}\right]=\left[\begin{array}{r}
0.1967 \\
-0.2607 \\
0.2356 \\
-0.0666 \\
0.0036 \\
0.3796 \\
0.3797 \\
-0.3355 \\
0.3474 \\
1.1750 \\
0.0009 \\
1.5913
\end{array}\right] ; \hat{\boldsymbol{\mu}}=\left[\begin{array}{l}
\mu_{1} \\
\mu_{2} \\
\mu_{3}
\end{array}\right]=\left[\begin{array}{l}
47.7816 \\
30.2635 \\
-3.0592
\end{array}\right]
$$

$$
\hat{\gamma}_{1}=\left[\begin{array}{l}
\gamma_{11} \\
\gamma_{21} \\
\gamma_{31}
\end{array}\right]=\left[\begin{array}{c}
-4.4505 \\
1.9577 \\
1.5276
\end{array}\right] ; \hat{\gamma}_{2}=\left[\begin{array}{l}
\gamma_{12} \\
\gamma_{22} \\
\gamma_{32}
\end{array}\right]=\left[\begin{array}{c}
4.4505 \\
-1.9577 \\
-1.5276
\end{array}\right] ;
$$

and the estimators equation models (25) are

$$
\begin{aligned}
\hat{y}_{1 i 1}= & 47.7816+0.1967 x_{11 i 1}-0.2607 x_{12 i 1} \\
& -1.6142 \mathbf{w}_{i}^{t} \mathbf{y}_{11}+0.3797 y_{2 i 1}-0.3355 y_{3 i 1} \\
& -4.4505+\hat{u}_{1 i 1} \\
\hat{y}_{2 i 1}= & 30.2635+0.2356 x_{21 i 1}-0.0666 x_{22 i 1} \\
& -1.6142 \mathbf{w}_{i}^{t} \mathbf{y}_{21}+0.3474 y_{1 i 1}+1.1750 y_{3 i 1} \\
& +1.9577+\hat{u}_{2 i 1} \\
\hat{y}_{3 i 1}= & -3.0592+0.0036 x_{31 i 1}+0.3796 x_{32 i 1} \\
& -1.5742 \mathbf{w}_{i}^{t} \mathbf{y}_{31}+0.0009 y_{1 i 1}+1.5913 y_{2 i 1} \\
& +1.5276+\hat{u}_{3 i 1}, \\
\hat{u}_{1 i 1}= & 0.9258 \mathbf{w}_{i}^{t} \hat{\mathbf{u}}_{11} \\
\hat{u}_{2 i 1}= & -0.2242 \mathbf{w}_{i}^{t} \hat{\mathbf{u}}_{21} \\
\hat{u}_{3 i 1}= & 0.4658 \mathbf{w}_{i}^{t} \hat{\mathbf{u}}_{31}, \\
\hat{y}_{1 i 2}= & 47.7816+0.1967 x_{11 i 2}-0.2607 x_{12 i 2} \\
& -1.6142 \mathbf{w}_{i}^{t} \mathbf{y}_{12}+0.3797 y_{2 i 2}-0.3355 y_{3 i 2} \\
& +4.4505+\hat{u}_{1 i 2} \\
\hat{y}_{2 i 2}= & 30.2635+0.2356 x_{21 i 2}-0.0666 x_{22 i 2} \\
& -1.6142 \mathbf{w}_{i}^{t} \mathbf{y}_{22}+0.3474 y_{1 i 2}+1.1750 y_{3 i 2} \\
& -1.9577+\hat{u}_{2 i 2} \\
\hat{y}_{3 i 2}= & -3.0592+0.0036 x_{31 i 2}+0.3796 x_{32 i 2} \\
& -1.5742 \mathbf{w}_{i}^{t} \mathbf{y}_{32}+0.0009 y_{1 i 2}+1.5913 y_{2 i 2} \\
& -1.5276+\hat{u}_{3 i 2}, \\
\hat{u}_{1 i 2}= & 0.9258 \mathbf{w}_{i}^{t} \hat{\mathbf{u}}_{12} \\
\hat{u}_{2 i 2}= & -0.2242 \mathbf{w}_{i}^{t} \hat{\mathbf{u}}_{22} \\
\hat{u}_{3 i 2}= & 0.4658 \mathbf{w}_{i}^{t} \hat{\mathbf{u}}_{32},
\end{aligned}
$$

where $\hat{\mathbf{u}}_{h j}$ are the estimate values for residual errors as given in Table 2.

\section{Conclusion}

In this paper, we are motivated to develop simultaneous equation models for fixed effect panel data with one-way error component by means of 3SLS solutions, especially for general spatial.

The numerical approximation estimators of parameter models are obtained by means of concentrated log-likelihood formulation with method of forming sequence. In this paper, we use the increasing values 0.01 .

The closed-form estimators are obtained by means of feasible generalized least squares-three- 
stage least squares (FGLS-3SLS) and they are called the estimators of feasible generalized least squaresmultivariate general spatial three-stage least squares fixed effect panel simultaneous models (FGLSMGS3SLSFEPSM). All estimators are consistent estimators.

There is one limitation of this paper, we still use the numerical approximation to find the estimators of spatial autoregressive and spatial autocorrelation. In future research, we encourage to find the closedform estimators of spatial autoregressive and spatial autocorrelation. In addition, to develop models not only for fixed effect but also both fixed effect and random effect (mixed models).

\section{References:}

[1] Sirait, T., Sumertajaya, I. M., Mangku, I. W., Asra, A. and Siregar, H., Multivariate spatial error three-stage least squares fixed effect panel: simultaneous models and estimation of their parameters, Far East Journal of Mathematical Sciences (FJMS) 102(12), 2017b, pp. 2941-2970.

[2] Sirait, T., Multivariate spatial autoregressive three-stage least squares fixed effect panel simultaneous models and estimation of their parameters, WSEAS Transactions on Mathematics 18, 2019, pp. 307-318.

[3] Koutsoyiannis, A., Theory of Econometrics, 1st ed., The MacMillan Press Ltd., London, 1973.

[4] Greene, W. H., Econometric Analysis, 7th ed., Pearson Education, Inc., Boston, 2012.

[5] LeSage, J. P., The Theory and Practice of Spatial Econometrics, Department of Economics. Toledo University, 1999.

[6] Anselin, L. and Kelejian, H. H., Testing for spatial error autocorrelation in the presence of endogenous regressors, International Regional Science Review 20, 1997, pp. 153-182.

[7] Tłuczak, A., The analysis of the phenomenon of spatial autocorrelation of indices of agricultural output, Quantitative Methods in Economics 14(2), 2013, pp. 261-271.

[8] Zhang, T. and Lin, G., A decomposition of Moran's I for clustering detection, Comput. Statist. Data Anal. 51, 2007, pp. 6123-6137.

[9] Baltagi, B. H. and Deng, Y., EC3SLS estimator for a simultaneous system of spatial autoregressive equations with random effects, Econometric Reviews 34, 2015, pp. 659-694.

[10] Krishnapillai, S. and Kinnucan, H., The impact of automobile production on the growth of nonfarm proprietor densities in Alabama's counties, Journal of Economic Development 37(3), 2012, pp. 25-46.

[11] Anselin, L., Spatial Econometrics: Methods and Models, Dordrecht, Kluwer Academic Publishers, The Netherlands, 1988.

[12] Jiang, J., Linear and Generalized Linear Mixed Models and Their Applications, Springer, New York, 2007.

[13] Myers, R. H. and Milton, J. S., A First Course In The Theory of Linear Statistical Models, PWS-KENT Publishing Company, Boston 1991.

[14] Goldberger, A. S., Econometric Theory, John Wiley \& Sons, Inc., New York, 1964.

[15] Kariya, T. and Kurata, H., Generalized Least Squares, John Wiley \& Sons, Ltd., England, 2004.

[16] Mood, A.M., Graybill, F.A. and Boes, D.C., Introduction to the Theory of Statistics, 3rd ed., McGraw-Hill, Inc., Auckland, 1974.

[17] Sirait, T., Sumertajaya, I. M., Mangku, I. W., Asra, A. and Siregar, H., Multivariate threestage least squares fixed effect panel simultaneous models and estimation of their parameters, Far East Journal of Mathematical Sciences (FJMS) 102(7), 2017a, pp. 1503-1521.

[18] Sirait, T., Sumertajaya, I. M., Mangku, I. W., Asra, A. and Siregar, H., Simultaneous Equation Models for Spatial Panel Data with Application to Klein's Model, Dissertation. Bogor Agricultural University, 2018.

[19] Gujarati, D. N. and Porter, D. C., Basic Econometrics, 5th ed., The McGraw-Hill Companies, Inc., New York, 2009.

[20] Anselin, L., Bera, A. K., Florax, R. and Yoon, M. J., Simple diagnostic test for spatial dependence, Regional Science and Urban Economics 26, 1996, pp. 77-104.

[21] Christ, C. F., Econometric Models and Methods, John Wiley \& Sons, Inc., New York, 1966.

[22] Dhrymes, P. J., Econometrics: Statistical Foundations and Applications, Springer-Verlag New York Inc., USA, 1974.

[23] Klein, L. R., Textbook of Econometrics, 2nd ed., Prentice-Hall, Inc., New Jersey, 1972.

\section{Creative Commons Attribution License 4.0 (Attribution 4.0 International, CC BY 4.0)}

This article is published under the terms of the Creative Commons Attribution License 4.0 https://creativecommons.org/licenses/by/4.0/deed.en US 